\title{
Gene expression analysis of hepatic roles in cause and development of diabetes in Goto-Kakizaki rats
}

\author{
Richard R Almon ${ }^{1,2,3}$, Debra C DuBois ${ }^{1,2}$, William Lai ${ }^{1}$, Bai Xue ${ }^{1}$, Jing Nie ${ }^{1}$ and William J Jusko ${ }^{2,3}$ \\ Departments of ${ }^{1}$ Biological Sciences and ${ }^{2}$ Pharmaceutical Sciences, State University of New York at Buffalo, 107 Hochstetter Hall, Buffalo, \\ New York 14260, USA \\ ${ }^{3}$ New York State Center of Excellence in Bioinformatics and Life Sciences, Buffalo, New York, 14203 USA \\ (Correspondence should be addressed to R R Almon; Email: almon@eng.buffalo.edu)
}

\begin{abstract}
Progression of diabetes was studied in male Goto-Kakizaki (GK) spontaneously diabetic rats between 4 and 20 weeks of age, and compared with Wistar-Kyoto (WKY) controls. Five animals from each strain were killed at $4,8,12,16$, and 20 weeks of age. Body weight, plasma glucose, and plasma insulin were measured. WKY rats showed a significantly larger weight gain than GK animals from 8 weeks of age onward. Plasma glucose was relatively stable in WKY. By contrast, plasma glucose was higher in GK than WKY even at 4 weeks and continued to increase up to 12 weeks and then maintained a hyperglycemic plateau throughout the remainder of the experiment. Plasma insulin was relatively stable in WKY from 8 weeks onward but was sharply elevated in GK between 4 and 8 weeks. After 8 weeks,
\end{abstract}

insulin declined in GK with GK concentrations lower than WKY at 20 weeks, suggesting $\beta$-cell failure. Gene expression in liver was explored using Affymetrix 230-2 gene arrays. Data mining identified 395 probe sets out of more than 31000 that were differentially regulated. Excluding unidentifiable probe sets and considering duplicate probe sets, there were 311 genes that were expressed differently in the liver of the two strains. A functional analysis of these genes indicated that disruption of lipid metabolism in the liver is a major consequence of the chronic hyperglycemia in the GK strain. In addition, the results suggest that chronic inflammation contributes significantly to the development of diabetes in the GK rats.

Journal of Endocrinology (2009) 200, 331-346

\section{Introduction}

Although rare forms of human type 2 diabetes are associated with defects in individual genes, more common forms appear to involve a complex interaction between predisposing genetic backgrounds and environmental factors. While epigenetic factors such as fetal programming may contribute, major aspects of the development of type 2 diabetes appear to result from interactions between the expression and/or structure of multiple genes with environmental lifestyle factors. The hallmark of type 2 diabetes is peripheral insulin resistance; however, the associated phenotypes in humans are quite heterogeneous. Other characteristics that may be, but are not always, associated with this form of diabetes are central obesity, dyslipidemia, hypertension, abnormal insulin secretion in response to glucose, hyperphagia, and excessive hepatic gluconeogenesis. At present, it is not clear the degree to which the various sub-phenotypes of human type 2 diabetes are the result of genetics, epigenetics, or environment (Sedova et al. 2007).

Several animal models have been developed for the study of type 2 diabetes. Many of these models such as the leptin-deficient (ob/ob) mouse, the leptin receptor-deficient $(\mathrm{db} / \mathrm{db})$ mouse, and the leptin receptor-deficient $(\mathrm{fa} / \mathrm{fa})$ rat are, like rare forms of the human type 2 disease, the result of single-gene defects. In fact, there are a large number of genes that can either be knocked out or overexpressed to cause an insulin-resistant phenotype (Almon et al. 2005a). In contrast to the single-gene defect models, Goto-Kakizaki (GK) rats are a polygenic diabetic rat model. GK rats have been widely used as a surrogate in type 2 diabetes studies. Originally developed in the early 1970s in Japan from multiple-generation breeding of Wistar-Kyoto (WKY) rats selected for high population values of glucose intolerance, these rats exhibit a spontaneous form of diabetes (O'Rourke et al. 1997, Portha 2005). Although many conflicting reports exist in the literature as to other characteristics of GK rats, elevated blood glucose, peripheral insulin resistance, and a nonobese phenotype are consistent features of this animal model. While no single animal model is a perfect surrogate for the human disease, the polygenic nature of inheritance and the previously demonstrated insulin resistance affecting muscle, adipose tissue, and liver in GK rats makes them a valid model for the human disease. However, since obesity is a factor in some but not all type 2 diabetic humans, the GK rat model is similar to only a subset of human type 2 diabetic patients in this regard (Prando et al. 1998).

The liver plays a central role in systemic energy metabolism and therefore is important in diseases such as diabetes where 
systemic energy substrate flow is altered. Liver is the only tissue that stores glucose in the form of glycogen; this glucose can be released in response to glucagon or epinephrine to maintain systemic concentrations. In addition to glucose storage and release, liver can also synthesize glucose de novo through the process of gluconeogenesis. In addition to carbohydrate metabolism, the liver is central to whole body lipid metabolism. About one-half of the cholesterol in the body is produced in the liver, and the liver is also central to the control, storage, and distribution of apolipoproteins.

The contributing role of the liver to hyperglycemia appears to be a variable in human type 2 diabetes. There is clear evidence that in some, but not all, cases, excessive gluconeogenesis in the liver contributes to hyperglycemia (Gastaldelli et al. 2000, Cohen \& Goedert 2004). Conversely, it is clear that chronically elevated blood glucose also has a significant impact on the liver itself. In contrast to skeletal muscle that primarily obtains glucose through the insulindependent GLUT4 transporter, the liver takes up glucose through the non-insulin-dependent GLUT2 (Bloomgarden 2003). As a result, uptake of glucose into the liver is highly controlled by the blood glucose concentration. Therefore, chronic hyperglycemia causes the liver to take up large amounts of glucose. Other than energy generation, the only two ways of using this glucose are glycogen synthesis and lipid synthesis. There are reports of excessive glycogen deposition in the liver associated with human diabetes (Vallance-Owen 1952, Clore et al. 1992). Much more common is the excessive buildup of fat in the liver (steatosis; Lonardo et al. 2005).

In the present study, we sought to carefully characterize disease progression (e.g., blood glucose and insulin) in the taconic colony of GK rats from shortly after birth through mid-adulthood. To that end, groups of GK and WKY rats were maintained in our facility from weaning through 20 weeks of age, with subgroups killed at $4,8,12,16$, and 20 weeks. Livers were removed from these animals and used for an extensive time-series analysis of gene expression using Affymetrix 230-2 gene arrays that contain more than 31000 probe sets. The results provide not only indications as to the cause of diabetes in these animals but also changes in liver gene expression that are the consequence of chronic hyperglycemia.

\section{Materials and Methods}

\section{Experimental design}

This study involved 30 GK spontaneously diabetic and 30 WKY control male rats obtained from Taconic Farms (Germantown, NY, USA). Our research protocol adheres to the 'Principles of Laboratory Animal Care' (NIH publication 85-23, revised in 1985) and was approved by the University at Buffalo Institutional Animal Care and Use Committee. Animals were received at $21 \pm 3$ days of age. For experimental purposes, all animals were considered 22 days old at the time of arrival. Rats were maintained in a separate room in our animal facilities under stringent environmental conditions that included strict adherence to $12 \mathrm{~h}$ light: $12 \mathrm{~h}$ darkness cycles. All animal manipulations and animal care were carried out between 1.5 and $3.5 \mathrm{~h}$ after lights on. Animals were housed in individual cages and body weights measured twice weekly on all animals. Five animals from each strain were killed at five different ages: 4, 8, 12, 16, and 20 weeks. At the time of killing, animals were anesthetized with ketamine $(80 \mathrm{mg} / \mathrm{kg}) /$ valium $(5 \mathrm{mg} / \mathrm{kg})$ i.p. Blood glucose was measured from whole blood at the time of killing. Animals were killed by aortic exsanguination using EDTA (4 mM final concentration) as anticoagulant. Plasma was prepared from blood by centrifugation $\left(2000 \mathrm{~g}, 4{ }^{\circ} \mathrm{C}\right.$, $15 \mathrm{~min}$ ), aliquoted, and stored at $-80^{\circ} \mathrm{C}$. Liver was harvested, weighed, rapidly frozen in liquid nitrogen, and stored at $-80{ }^{\circ} \mathrm{C}$ for future studies.

\section{Assays}

Blood glucose Blood glucose was measured from whole blood at the time of killing using a BD Logic blood glucose meter (BD Medical, Franklin Lakes, NJ, USA).

Plasma glucose Plasma glucose was measured by the glucose oxidase method (Sigma GAGO-20). The manufacturer's instructions were modified such that the assay was carried out in a $1 \mathrm{ml}$ assay volume, and a standard curve consisting of seven concentrations over a 16-fold range was prepared from the glucose standard and run with each experimental set. Experimental samples were run in triplicate.

\section{Plasma insulin}

Insulin was measured in plasma samples using a commercial RIA (RI-13K Rat Insulin RIA Kit, Millipore Corporation, St Charles, MO, USA). All assays were carried out according to the manufacturer's directions with standards run in duplicate and experimental samples run in triplicate.

\section{Liver triglycerides}

Frozen liver samples were extracted in chloroform:methanol $(2: 1)$, dried, and resuspended in butanol as described previously (Hundal et al. 2000). Extracted triglycerides were assayed colorimetrically with commercial reagents (WAKO Chemicals, Richmond, VA, USA).

\section{Statistical analysis}

For statistical comparisons, two-way ANOVAs were carried out using SigmaStat 3.5 software (Systat Software, Point Richmond, CA, USA) with Tukey post hoc tests. Since data were not normally distributed, ANOVAs were performed on rank-transformed data.

\section{Microarrays}

Liver samples from each animal were ground to a fine powder in a mortar cooled by liquid nitrogen and $100 \mathrm{mg}$ was added 
to $1 \mathrm{ml}$ pre-chilled TRIzol reagent (Invitrogen, Carlsbad, CA, USA). Total RNA extractions were carried out according to the manufacturer's directions and were further purified by passage through RNeasy mini-columns (Qiagen, Valenvia, CA, USA) according to the manufacturer's protocols for RNA cleanup. Final RNA preparations were resuspended in RNase-free water and stored at $-80{ }^{\circ} \mathrm{C}$. The RNAs were quantified spectrophotometrically, and purity and integrity assessed by agarose gel electrophoresis. All samples exhibited 260/280 absorbance ratios of approximately $2 \cdot 0$, and all showed intact ribosomal $28 \mathrm{~S}$ and $18 \mathrm{~S}$ RNA bands in an approximate ratio of 2:1 as visualized by ethidium bromide staining. Isolated RNA from each liver sample was used to prepare target according to the manufacturer's protocols. The biotinylated cRNAs were hybridized to 50 individual Affymetrix GeneChips Rat Genome 230-2 (Affymetrix, Inc., Santa Clara, CA, USA), which contained more than 31000 probe sets.

\section{Data mining}

Affymetrix Microarray Suite 5.0 (Affymetrix) was used for initial data acquisition and analysis. The signal intensities were normalized for each chip using a distribution of all genes around the 50th percentile. The dataset was then loaded into a data mining program, GeneSpring 7 (Silicon Genetics, Redwood City, CA, USA), for further analysis. The generated dataset was submitted to the National Center for Biotechnology Information (NCBI) Gene Expression Omnibus (GEO; http://www.ncbi.nlm.nih.gov/projects/geo/) database (GSE 13271). In order to screen for probe sets with different expression levels in the GK versus WKY, each probe set on the five GK chips at each time point was normalized to the average of the same probe set on the five WKY chips. Thus, if there is no difference between GK and WKY the result is a value of 1. If GK is greater than WKY the value is greater than 1 , and if GK is less than WKY, then the value is less than 1.

In order to objectively identify probe sets of interest, the entire dataset was filtered with criteria similar to the ones applied to previous gene array datasets (Almon et al. 2005b, $2007 a, b)$. This approach does not select for probe sets but rather eliminates those probe sets that do not meet certain criteria, leaving the remainder for further consideration. The first filter was designed to eliminate probe sets for genes that are not expressed in the liver. This filter employed a function in the Affymetrix Microarray Suite 5.0. During the initial data analysis, a 'call' of present (P), absent (A), or marginal (M) for each probe set on each chip was determined based on the intensity comparison of the matched and mismatched probe sequence pairs. The first filter eliminated all probe sets that did not have a call of P on at least five of the GK chips. Starting with 31099 probe sets this filter eliminated 13460 probe sets leaving 17639 probe sets for further consideration. We repeated this filter, but in this case normalizing the WKY to the average of each probe set for the GK. This yielded an additional 708 probe sets not on the list when GK was normalized to WKY. The reversal and refiltering considered the possibility that a probe set could be absent on the chips of one strain but expressed on the chips of the other strain. This filtering step left a remainder of 18347 probe sets to be carried forward into the next step.

The second level of filtering eliminated probe sets that could not meet the basic criterion of having different expression levels in GK and WKY rats. We employed two filters that eliminated probe sets that were neither lower nor higher in GK relative to WKY. The first filter started with the 5P-filtered list to remove all probe sets that did not have average values below 0.5 for at least three time points. Those probe sets retained thus had expression levels in which WKY is at least twice that of GK. The next filter again started with the 5P-filtered list, and eliminated all probe sets that did not have average values above 2 for at least three time points. Those probe sets retained by this filter thus had expression levels at least twice as high in GK relative to WKY.

\section{Quantitative real-time RT-PCR}

Because gluconeogenesis in the liver contributes to hyperglycemia in many humans with type 2 diabetes, the array results for the enzyme phosphoenolpyruvate carboxykinase (PCK1) were confirmed by real-time quantitative RT-PCR. PCK1 is the ratelimiting enzyme in gluconeogenesis. The quantity of liver Pck1 mRNA along with gene-specific in vitro-transcribed cRNA standards was determined by quantitative real-time RT-PCR using TaqMan probes. Briefly, primer and probe sequences were designed using PrimerExpress software (Applied Biosystems, Foster City, CA, USA) and custom synthesized by Biosearch Technologies, Inc. (Novato, CA, USA). The RT-PCR was performed using Brilliant QRT-PCR Core Reagent Kit, 1-Step (Stratagene, La Jolla, CA, USA) in a Stratagene MX3005P thermocycler according to the manufacturer's instructions. A standard curve was generated using the in vitro-transcribed sense cRNA standards. Primer and probe sequences are as follows: forward primer (5'-CCGGGCACCTCAGTGAAG-3'), reverse primer $\left(5^{\prime}\right.$-CACGTTGGTGAAGATGGTGTTT-3 $\left.{ }^{\prime}\right)$, probe $\left(5^{\prime}\right.$-FAM-ATCCGAACGCCATTAAGACCATCBHQ- $\left.3^{\prime}\right)$. Additional minus reverse transcriptase controls were run for each RNA sample analyzed; all controls exhibited lack of amplification. Intra- and interassay coefficient of variation values were less than $15 \%$.

\section{Results}

\section{Animals}

Figure 1 presents mean body weights of all animals throughout the course of this study. As expected, both strains of animals exhibited continual increases in body weight throughout the study period. Significant differences in strain were present from 8 weeks of age onward. WKY animals were heavier than GK animals by 8 weeks $(P=0 \cdot 004)$, and the 


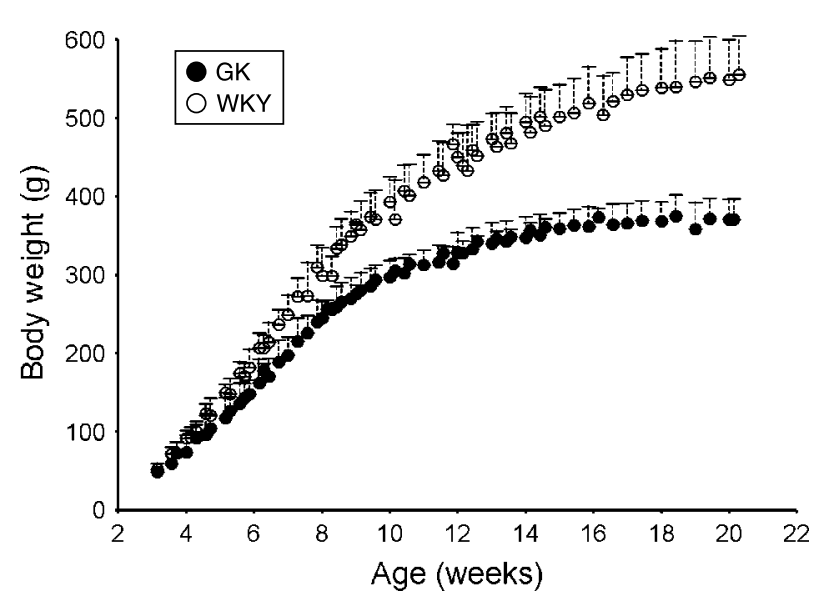

Figure 1 Body weights of GK and WKY rats from 3 to 20 weeks of age. Symbols represent means and error bars 1 s.D. of the mean. Closed circles, GK; open circles, WKY.

disparity in weight became more pronounced at later ages $(P<0 \cdot 001)$.

Figure 2 (top panel) shows the change in absolute liver weight as a function of time. There is a large increase in the weight of the livers in both GK and WKY between 4 and 8 weeks followed by slow asymptotic increases over 20 weeks. Figure 2 (bottom panel) shows liver weight as a percentage of total body weight. By 8 weeks and continuing throughout the remainder of the experiment, the liver was a significantly larger percentage of total body weight in GK when compared with WKY. Measurement of liver triglycerides showed slight age-dependent increases, but no statistical differences between GK and WKY animals at any age (data not shown). Glucose was measured in whole blood at the time of killing using a glucometer. However, at later ages, many GK rats had blood glucose values that exceeded the range of the meter $(600 \mathrm{mg} / \mathrm{dl})$. Therefore, glucose concentrations were also measured in plasma by an enzymatic assay. The ratio of individual values of blood glucose:plasma glucose had a mean of $1 \cdot 03 \pm 0 \cdot 16$, and the correlation between the two values was $0 \cdot 89$. Figure 3 presents mean plasma glucose values at the time of killing.

Plasma glucose concentrations increased with age in both strains, reaching an apparent plateau between 8 and 12 weeks of age. Not unexpectedly, GK animals had higher plasma glucose compared with control WKY animals at all ages $(P<0.001$ at all ages). Plasma insulin determined from RIA analyses is presented in Fig. 4. Variation between animals within a group exhibited high S.D. This high variability was likely due to true animal variation rather than experimental error, since inter-assay variability averaged $4 \cdot 3 \pm 3 \cdot 3 \%$ for all samples, and variability of quality control samples (intra-assay variability) was under $6 \%$. In addition, the samples were also analyzed by an ELISA method, which, despite higher inter-assay variability in our hands, resulted in almost identical insulin profiles (data not shown). The general trend in the data appears to indicate an increase in plasma insulin between 4 and 8 weeks of age in both strains, with GK generally higher
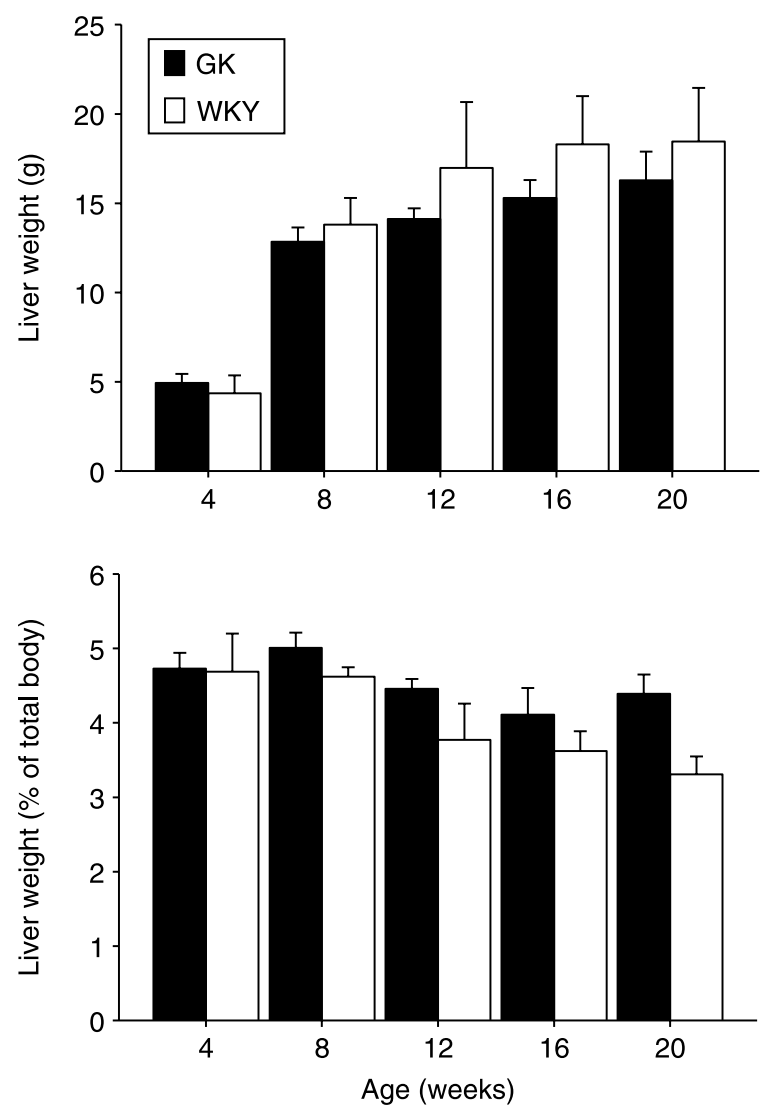

Figure 2 Mean liver weights of GK and WKY rats as a function of age. Top panel represents weights of liver in grams, and bottom panel liver weights as a percentage of animal body weight. Error bars represent 1 S.D. of means.

than WKY values between 8 and 16 weeks. However, at 20 weeks of age, GK values appear lower than WKY controls. These strains tested as significantly different $(P$ at least $<0 \cdot 05)$ at all ages except 16 weeks.



Figure 3 Plasma glucose concentrations of GK and WKY rats as a function of age. Error bars represent 1 s.D. of means. 


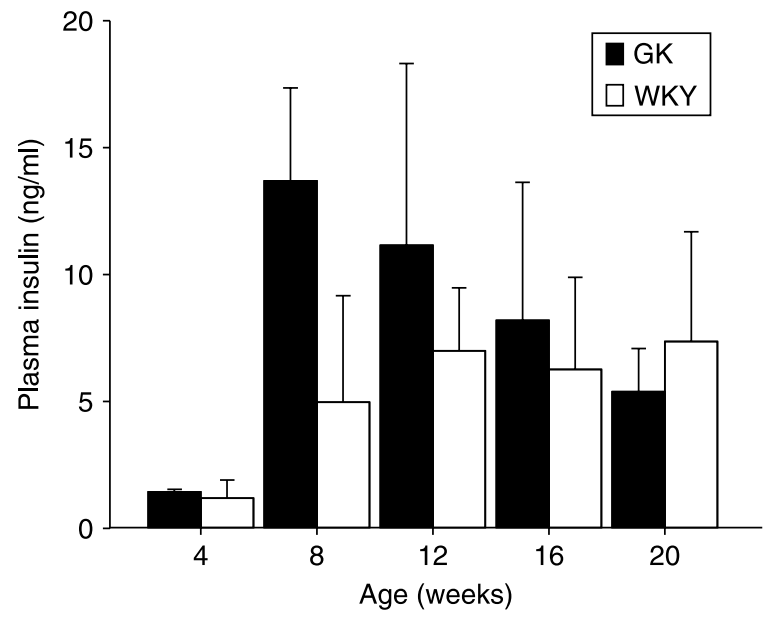

Figure 4 Plasma insulin concentrations of GK and WKY rats as a function of age. Error bars represent 1 s.D. of means.

\section{Data mining}

The Affymetrix R230-2 chip contains more than 31000 probe sets. The initial problem is to identify probe sets of interest for further attention. Because the chip can measure an expression range of over three to four orders of magnitude, the first problem is to bring all probe sets into a common range. To accomplish this, we divided the signal for each probe set on each chip for GK by the average of that probe set on the five WKY chips. The result is that no difference yields a value of 1 . If GK is greater than WKY, the value is greater than 1, and if WKY is greater than GK, the value is less than 1. Using this normalized dataset, we eliminated probe sets that were not expressed in the liver by filtering using the Affymetrix call flag. Previously, we used a similar filtering as an approach to eliminate probe sets not expressed in a tissue (liver, muscle, and kidney) in previous Affymetrix gene array time-series datasets (Almon et al. 2005b, $2007 a, b)$. Eliminating probe sets that did not get a call of P on at least five chips on either GK or WKY left a remainder of 18347 probe sets. We then eliminated probe sets in which the expression was not different in GK and WKY. We sought to identify two types of probe sets. The first were probe sets for genes that are causative to the difference in physiology between GK and WKY. Our assumption was that the expression of these genes should be different at all five ages. The second were probe sets that reflect the consequence of chronic hyperglycemia in GK rats. These should correspond to the development of hyperglycemia in GK and should be different in at least three time points. We therefore chose to retain probe sets different at three, four, or five time points. Elimination of all probe sets in which GK were not twice as high as WKY at three to five time points left a remainder of 174 probe sets. Conversely, eliminating all probe sets in which WKY were not at least twice as high as GK at three to five time points left a remainder of 221 probe sets. Together, the two lists yielded 395 probe sets for further consideration. Figure 5 shows a gene tree of all probe sets on the 230-2 chip (left panel) compared with the gene tree of the 395 probe sets not eliminated by the filters (right panel). In this presentation, the probe sets are displayed vertically as a tree and their expression dynamics over time are displayed horizontally in colors with yellow in the graph representing an expression ratio around 1 or no difference. Colors progressing toward red indicate normalized values greater than 1 (GK greater than WKY by at least twofold), and colors toward blue indicate values less than 1 (WKY greater than GK by at least twofold). The brightness of the colors reflects the original signal intensities or expression levels before normalization. The more abundantly expressed mRNAs exhibit brighter colors. The $x$-axis provides the five ages studied. The $y$-axis represents the list of the probe sets in order of similarity. Supplementary Table 1, see Supplementary data in the online version of the Journal of Endocrinology at http://joe.endocrinology-journals.org/content/vol200/issue3/ provides raw data for each of the 395 probe sets in all 50 samples.

\section{Data analysis}

Affymetrix provides an accession number for the sequence from which each probe set was built. We submitted the accession number of each selected probe set to the NCBI Basic Local Alignment Search Tool (BLAST) to identify, as closely as possible, its gene. Of the 395 probe sets, there were 55 probe sets that could not be identified by the BLAST of their accession numbers. In addition, in several instances, there were multiple probe sets for the same gene. This left 306 individual genes. These genes were then submitted to NCBI 'across database search' primarily to identify all aliases and alternate symbols. The preferred symbol was submitted to NCBI AceView as well as extensive PubMed Boolean logic searches to ascertain the function of the gene in liver. Based on these searches, we separated the genes into groups based on their function in the liver. These groups were as follows: transcription/translation; signaling; protein processing; lipid metabolism; immune/inflammatory; small molecule metabolism; cell cycle control; mitochondrial; and transport. An additional 24 genes that did not readily fit into these categories were grouped as miscellaneous, and the 55 probe sets not identifiable by BLAST were listed as ESTs. Clearly, such functional categorizations are not perfect because overlaps exist. For example, a transcription factor such as SREPB that is central to the regulation of lipid metabolism would be placed in lipid metabolism not transcription/translation, or a cytokine placed in the immune/inflammatory group rather than in the signaling group. Supplementary Table 2, see Supplementary data in the online version of the Journal of Endocrinology at http://joe. endocrinology-journals.org/content/vol200/issue3/ provides a list of all differentially regulated genes organized by these functional categories, and includes their probe set ID, accession number, gene name, symbol, and which strain was higher in how many conditions (ages). The functional analysis indicated that the genes that are differentially expressed in the GK and WKY strains are heavily weighted towards four functions. These functional groups are immune/inflammatory (67 probe sets), 




Figure 5 Gene tree representations of probe sets on individual Affymetrix R230-2 gene chips hybridized to total RNA prepared from the livers taken from GK and WKY rats as a function of age. The left panel represents all probe sets on the chip, and the right panel represents the 395 probe sets identified as differentially regulated in GK compared with WKY animals. The $x$-axis represents the age of animals at killing. The $y$-axis represents the list of probe sets grouped by similarity using Pearson's correlation. The yellow color indicates no change from controls, red indicates probe sets with enhanced expression relative to controls, and blue indicates suppressed expression relative to controls.

signal transduction (48 probe sets), transcription/translation (57 probe sets), and lipid metabolism (46 probe sets).

Normalization of GK to WKY and WKY to GK provided the ratios we used in the gene selection process. Once genes were selected, we restructured the dataset to directly examine the basis for the difference in the ratio. For example, the ratio data for probe set 1377672 showed that with increasing age there is steadily increasing difference in the ratio, with WKY $>$ GK. The direct comparison (Fig. 9B) demonstrates that the expression is increasing in WKY while staying essentially constant in GK. The change in the ratio could just as plausibly been due to WKY remaining constant while GK declined. Using this direct view, we were able to gain insight into both the underlying cause of the diabetes in the GK strain and the gene expression consequences of chronic hyperglycemia.

\section{Immune/inflammatory processes}

This functional category of differentially regulated genes (67 probe sets) indicates a generally altered inflammatory state in GK animals. Out of the 67 probe sets, 45 were higher in WKY when compared with GK animals, while 22 were higher in GK when compared with WKY. A large number of these genes are involved in antigen processing and presentation, while many others are involved in cytokine/chemokine signaling pathways (Supplementary Table 2). Selected examples of genes in this category are presented in Fig. 6. Figure 6A shows the expression profile of CD14 antigen (Cd14). CD14 is an initiator of the natural immunity signaling cascade responsible for the induction of the inflammatory response caused by bacterial cell wall lipopolysaccharide (LPS; Kubota et al. 2004). The expression of Cd14 is much higher in the livers of diabetic GK rats when compared with WKY at all ages examined. Adding to the picture of chronic inflammation in the GK rats are the expression patterns of two interferon-induced genes: Fig. 6B, interferon-induced protein with tetratricopeptide repeats 1 (Ifit) and Fig. 6C, interferon-inducible GTPase (Iigp1; Boehm et al. 1998, Ogawa et al. 2005). Both of these interferon-induced genes are much higher in the livers of diabetic GK animals than in control WKY rats at all ages. Interferons (IFNs) are involved in natural anti-virus immunity. In addition to suggesting a heightened inflammatory state in these animals, excessive circulating concentrations of interferon are also associated with insulin resistance (Mofredj et al. 2002, Li et al. 2005). Another gene suggesting chronic inflammation in the GK rats is chemokine (C-X-C motif) ligand 14 (Cxcl14). Nara demonstrated that disruption of $\mathrm{Cxcl14}$ greatly reduced obesity-induced insulin resistance in mice, and that restoring the gene restored the insulin resistance (Nara et al. 2007). Figure 6D shows that Cxcl14 is chronically higher in GK when compared with WKY.

\section{Signal transduction}

Signal transduction is integral to both regulation of energy metabolism and immune modulation, and not unexpectedly many differentially regulated genes in GK liver are involved with 


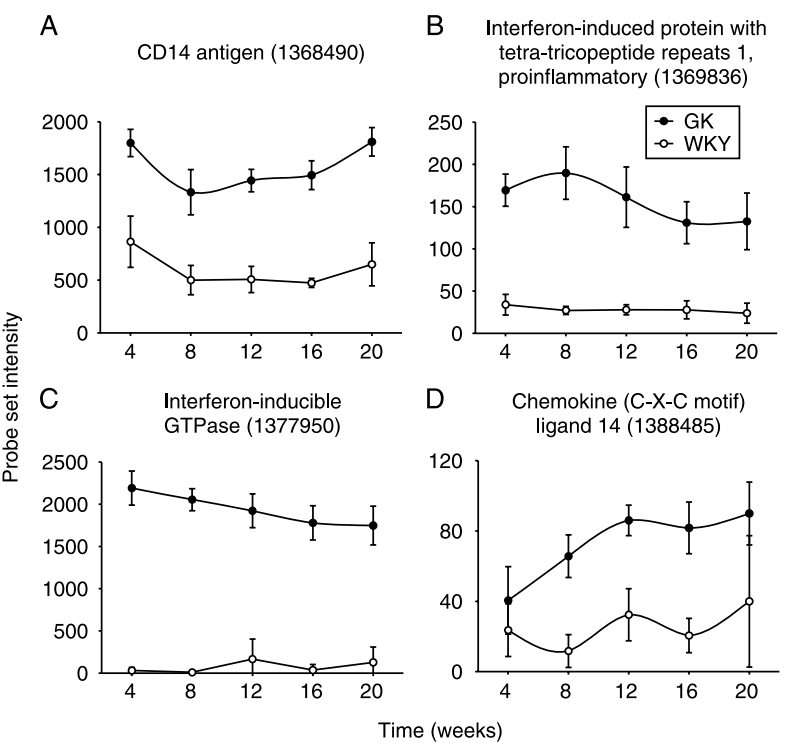

Figure 6 Examples of differentially regulated Immune/Inflammatory-related genes in GK and WKY rats as a function of age. The $y$-axis represents raw, non-normalized probe set intensities and the $x$-axis animal age. Symbols represent mean values of chip data from five animals and error bars 1 S.D. of the mean.

signal transduction pathways. Examples of genes in this functional grouping are presented in Fig. 7. Several of these are directly involved in or have been associated with insulin signaling. For example, the phosphatidylinositol 3-kinase (PI 3-kinase), regulatory subunit, polypeptide 1 (p85 alpha) (PIK3R1) pathway is one of two signaling cascades central to the actions of insulin on peripheral tissues including liver (Matsumoto et al. 2003). Alterations in the PIK3R 1 central hub of insulin metabolic actions have been linked to insulin resistance both through allelic variation and expression (Almind et al. 2002, Ueki et al. 2003). PIK3R1 is a heterodimer containing a regulatory subunit and a catalytic subunit. There are several isoforms of both the catalytic and regulatory subunits. Figure 7A and $\mathrm{B}$ reflects significant differences between GK and WKY in the expression of one of the regulatory isoforms and one of the catalytic isoforms. Figure 7A shows the profiles of PI 3-kinase, regulatory subunit, polypeptide 1 (p85 alpha; Pik3r1). The expression of Pik3r1 is higher in WKY than GK at all ages. Figure 7B shows the expression profiles of a catalytic subunit, phosphoinositide-3-kinase, class 3 (Pik3c3). Although at 4 weeks the message for this gene is more highly expressed in GK than WKY, starting at 8 weeks and for the remainder of the experiment, Pik3c3 message is more highly expressed in WKY than GK.

PFTAIRE protein kinase 1 (Pftk1), which is a cell cyclerelated kinase (Shu et al. 2007), is another gene whose expression is remarkably different in GK and WKY. As shown in Fig. 7C, the expression of Pftk1 is substantially higher in WKY when compared with GK, suggesting that cell proliferation may be reduced in the livers of GK rats The last gene in these examples is myotubularin-related protein 7

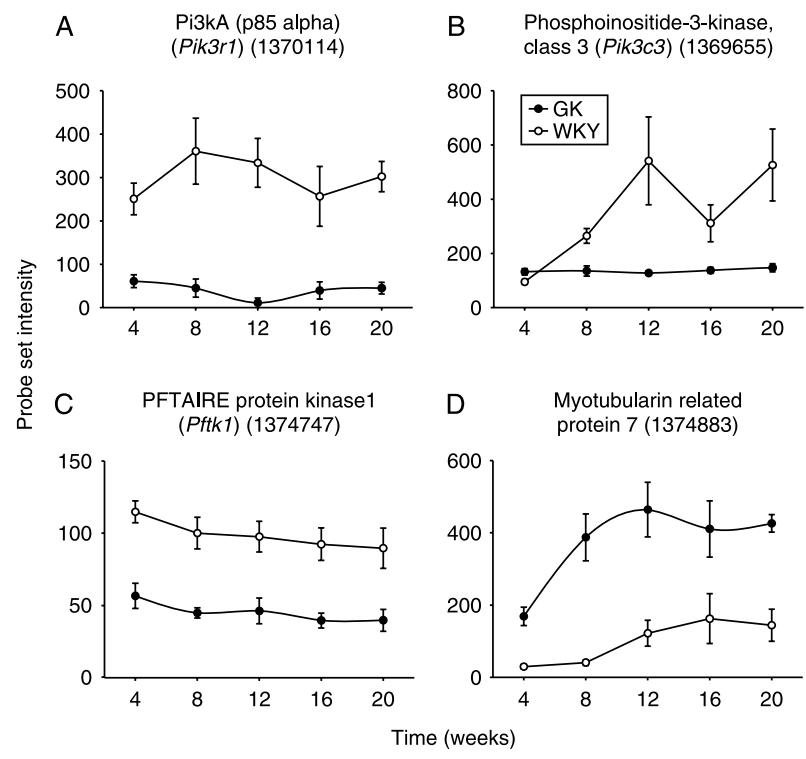

Figure 7 Examples of differentially regulated genes related to signal transduction in GK and WKY rats as a function of age. The $y$-axis represents raw, non-normalized probe set intensities and the $x$-axis animal age. Symbols represent mean values of chip data from five animals and error bars 1 S.D. of the mean.

(Mtmr7). MTMR7 has been shown to dephosphorylate PtdIns, a central component to insulin signaling (Mochizuki \& Majerus 2003). The much higher expression of this gene in GK relative to WKY may provide another mechanism of interference with insulin action (Fig. 7D).

\section{Transcription/translation}

This functional group contains a large number of differentially regulated genes. Many of these gene products are themselves controllers of gene expression (i.e., transcription factors). Many of these transcriptional regulators are associated with control of energy metabolism or inflammation. For example, signal transducer and activator of transcription 3 (STAT3) is a transcription factor that is involved is a variety of cellular functions. Employing knockout experiments, it has been observed that STAT3 plays a critical role in controlling inflammation and that deletion of Stat 3 causes an exacerbated inflammatory response (Kano et al. 2003). Figure 8A shows that Stat 3 expression is much higher in the livers from WKY when compared with GK animals. Figure $8 \mathrm{~B}$ presents expression patterns of crystallin, mu (Crym), a gene that plays pivotal roles in capturing and transporting thyroid hormone $\left(\mathrm{T}_{3}\right)$ into the nucleus (Suzuki et al. 2007). Thyroid hormone influences the expression of many genes that are also influenced by insulin. Figure $8 \mathrm{~B}$ shows that the expression of Crym is much lower in GK compared with WKY.

The third example, cellular repressor of E1A-stimulated genes 1 (CREG1), is a glycoprotein produced and secreted by the liver that binds to the insulin-like growth factor 2 receptor 


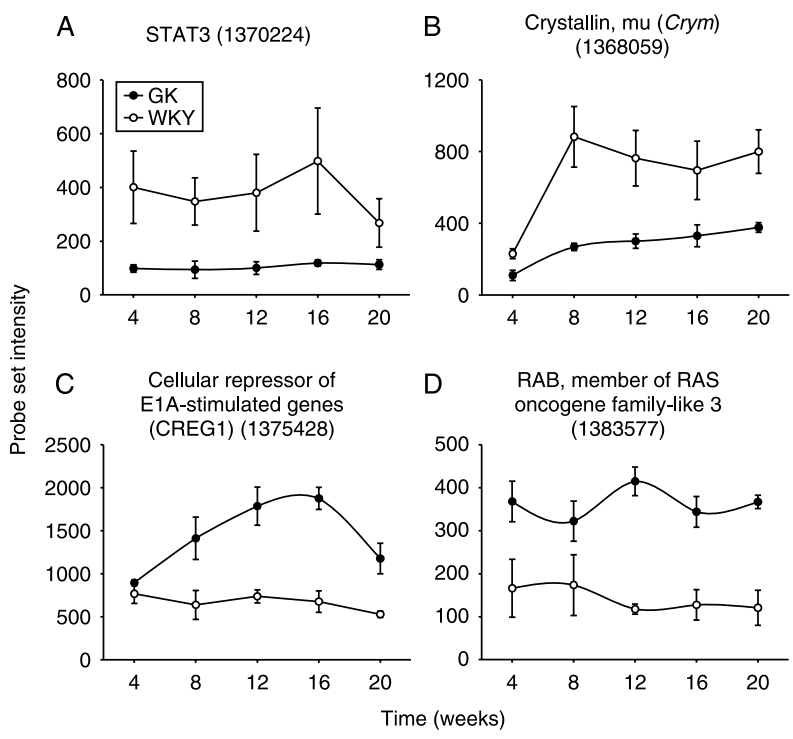

Figure 8 Examples of differentially regulated genes related to transcription/translation in GK and WKY rats as a function of age. The $y$-axis represents raw, non-normalized probe set intensities and the $x$-axis animal age. Bars represent mean values of chip data from five animals. Symbols represent mean values of chip data from five animals and error bars 1 s.D. of the mean.

and generally inhibits growth and thus energy expenditure by the animal (Bauer et al. 2004). CREG1 is strongly upregulated by starvation and calorie restriction (Bauer et al. 2004). Therefore, it is interesting that Creg1 is consistently more highly expressed in GK than WKY animals (Fig. 8C). Figure $8 \mathrm{D}$ presents the expression pattern of the transcription factor RAB. RAB, a member of RAS oncogene family-like 3 (Rabl3), participates in the regulation of genes involved in vesicular trafficking and its upregulation in the liver has been associated with obesity (Wilton \& Matthews 1996). The expression of Rabl3 is much higher at all ages in GK relative to WKY rats (Fig. 8D). The examples presented here further support the conclusion that there are alterations in insulin signaling capacity, altered energy metabolism, and altered immune responses in the livers from diabetic animals.

\section{Lipid metabolism}

Figure 9 provides examples of genes involved in various aspects of lipid metabolism that are differentially expressed in the GK and WKY strains. Figure 9A presents expression of cytoplasmic, epoxide hydrolase 2 (Ephx2). EPHX2 is an important enzyme in the metabolic breakdown of arachidonic acid-derived eicosanoids (Lee et al. 2006). Eicosanoids are involved in a variety of processes ranging from blood pressure regulation and calcium homeostasis to the systemic response to inflammation (De Caterina \& Basta 2001). Ephx2 is much more highly expressed in the livers from WKY than GK animals. Polymorphisms of EPHX2 are associated with

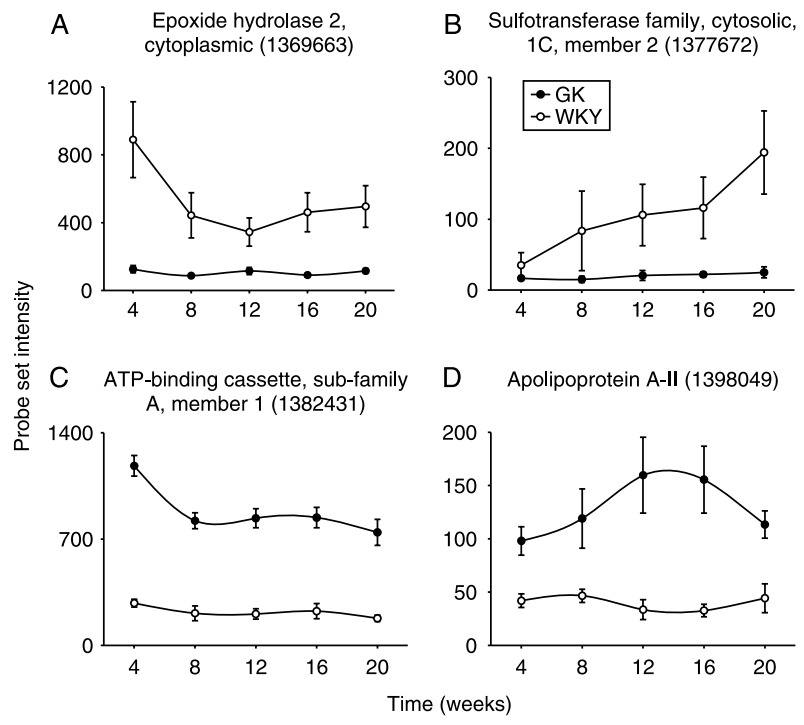

Figure 9 Examples of differentially regulated genes related to lipid metabolism in GK and WKY rats as a function of age. The $y$-axis represents raw, non-normalized probe set intensities and the $x$-axis animal age. Bars represent mean values of chip data from five animals. Symbols represent mean values of chip data from five animals and error bars 1 S.D. of the mean.

predispositions for a variety of dyslipidemic states such as hypercholesterolemia as well as hypertension and insulin resistance (Sato et al. 2004).

Figure 9B provides the expression profile for sulfotransferase family, cytosolic, 1C, member 2 (Sult1c2). SULT1C2 is involved in the sulfate conjugation of many endogenous and exogenous compounds. Of particular relevance to the liver is the degradation of bile acids (Vemula et al. 2004). It is also strongly downregulated as part of the complex series of reactions known as an acute phase response (Vemula et al. 2004). The livers from WKY animals show a continuous increase in expression of Sult1c2 with age. By contrast, this age-related enhancement fails to occur in GK rats. These data also support the hypothesis that chronic activation of the inflammation-related acute phase response is occurring in the GK animals.

Figure 9C provides the expression profiles of ATP-binding cassette, sub-family $\mathrm{A}$, member 1 (Abca1), which is a cholesterol efflux pump in the cellular lipid removal pathway. $A B C A 1$ controls the rate-limiting step in high-density lipoproteins (HDL) particle assembly by mediating efflux of cholesterol and phospholipids from cells to lipid-free $A P O A 1$, which forms nascent HDL particles (Tanaka et al. 2008). The expression of this gene is much higher in the livers from GK compared with WKY rats. Figure 9D shows the expression of apolipoprotein A-II (Apoa2). Apoa2 is also much higher in GK relative to WKY at all ages. $A P O A 2$ is the second most abundant apolipoprotein in HDL and has a role in the regulation of lecithin cholesterol acetyltransferase (LCAT). LCAT is the major enzyme involved in the esterification 
of free cholesterol present in circulating plasma lipoproteins and is a major determinant of plasma HDL concentrations (Weng \& Breslow 1996). These examples are not inclusive of all differentially expressed genes categorized as related to lipid metabolism (Supplementary Table 2). However, they are meant to illustrate that there are differences in the expression of genes involved in many aspects of lipid metabolism (bile acid, fatty acid, trigylceride, and cholesterol metabolism) in liver between GK and WKY rats.

\section{Other regulated genes of potential interest}

There are many additional genes that also warrant attention because of their differential expression in GK and WKY rats. The first two examples presented here are biliverdin reductase B (Blvre) and glutathione peroxidase 2 (Gpx2). The expression profiles for these two genes are shown in Fig. 10, and we have classified both under the functional grouping of Small Molecule Metabolism. Both BLVRB and GPX2 have antioxidant capabilities and are up regulated in response to oxidative stress (Wang \& Ortiz de Montellano 2003, Yan \& Chen 2006). Figure 10A and B demonstrates that both genes are more highly expressed at all ages in GK when compared with WKY. Another example is Resp 18. Although initially thought to be endocrine tissue specific, regulated endocrine-specific protein 18 (RESP18) has been found in many tissues including liver. This gene is involved in secretory processes and seems to have tissue-specific regulation (Bloomquist et al. 1994, Schiller et al. 1995).

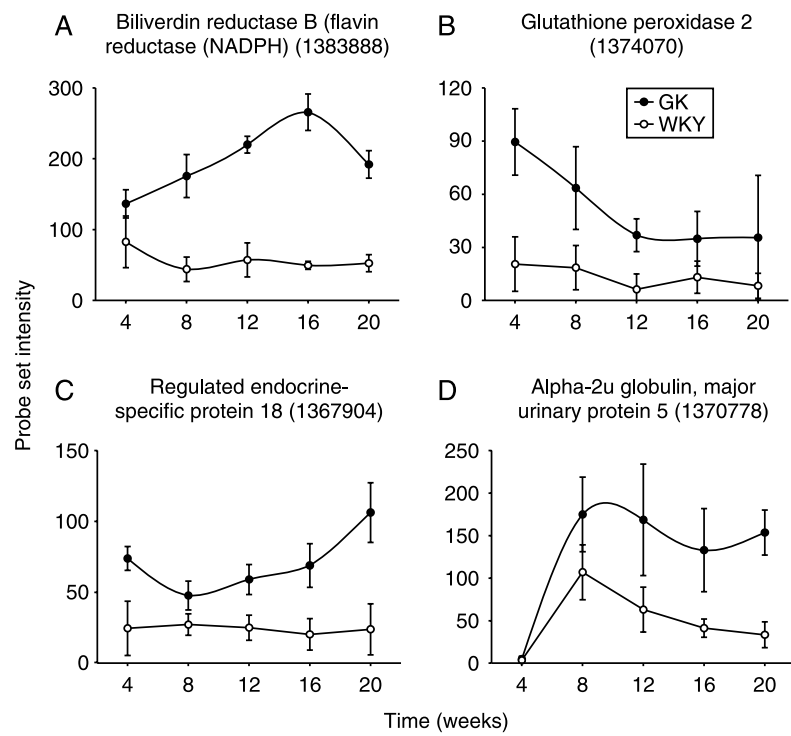

Figure 10 Examples of differentially regulated genes in other functional categories in GK and WKY rats as a function of age. The $y$-axis represents raw, non-normalized probe set intensities, and the $x$-axis animal age. Bars represent mean values of chip data from five animals. Symbols represent mean values of chip data from five animals and error bars 1 S.D. of the mean.
As shown in Fig. 10C, Resp 18 is more highly expressed in GK relative to WKY and that the difference seems to increase with age after 8 weeks. The last gene in these examples is one whose sequence blasts to sequences for $\alpha-2 u$ globulin and major urinary protein 5 (Mup5; Rowland et al. 2005). Although it is not clear exactly what this protein does and its relationship to diabetes, the profile of this lipid-binding protein is quite interesting. Figure 10D shows the expression profiles of this gene in both GK and WKY rats. At 4 weeks of age, the expression is hardly measurable in both strains. However, at 8 weeks, it is strongly expressed in both strains with GK higher than WKY. The difference between GK and WKY increases with age. Out of the 341 currently identifiable probe sets that are differentially expressed in the livers of diabetic animals, 219 are functionally grouped in one out of the four categories (immune/inflammatory, signaling, transcription/translation, or lipid metabolism).

However, an additional 122 probe sets were found to be regulated in our experiment, and the four profiles presented in Fig. 10 are simply examples of these. Raw data for all probe sets identified as differentially regulated can be found in Supplementary Table 1. In addition, the entire dataset is available through the NCBI GEO repository (GSE13271).

\section{Phosphoenolpyruvate carboxykinase 1 (PCK1)}

There are reports that in a subset of human type 2 diabetic patients, excessive gluconeogenesis by the liver significantly contributes to the hyperglycemia (Hundal et al. 2000). PCK1, which converts oxaloacetate into phosphoenolpyruvate and carbon dioxide, is the rate-limiting enzyme for gluconeogenesis. The array contained a probe set for PCK1 (1372264_at). Although this probe set did not meet our selection criteria of having a twofold difference in three out of five time points, we did examine the data directly. The array results indicated that GK was significantly higher than WKY $(P<0 \cdot 001)$ only at 4 weeks. We reexamined this result using QRT-PCR. The highly significant difference between GK and WKY at 4 weeks was confirmed by QRT-PCR. However, QRT-PCR did indicate that GK was significantly higher with a lesser $P$ value of $<0.05$ at three other times as well $(8,12$, and 20 weeks). The results, presented in Fig. 11, do not indicate a great difference between GK and WKY in the Pck1 expression except at 4 weeks when measured with either methodology. While in general agreement, the difference in results obtained by the two methods reflects the greater precision of QRT-PCR.

\section{Discussion}

The liver is the central hub in the flow of systemic energy substrates. In sedentary circumstances, about $75 \%$ of the blood entering the liver comes from the hepatic portal vein. Following eating, this blood rich in glucose and GLUT2 will mediate the uptake of large quantities of glucose into 

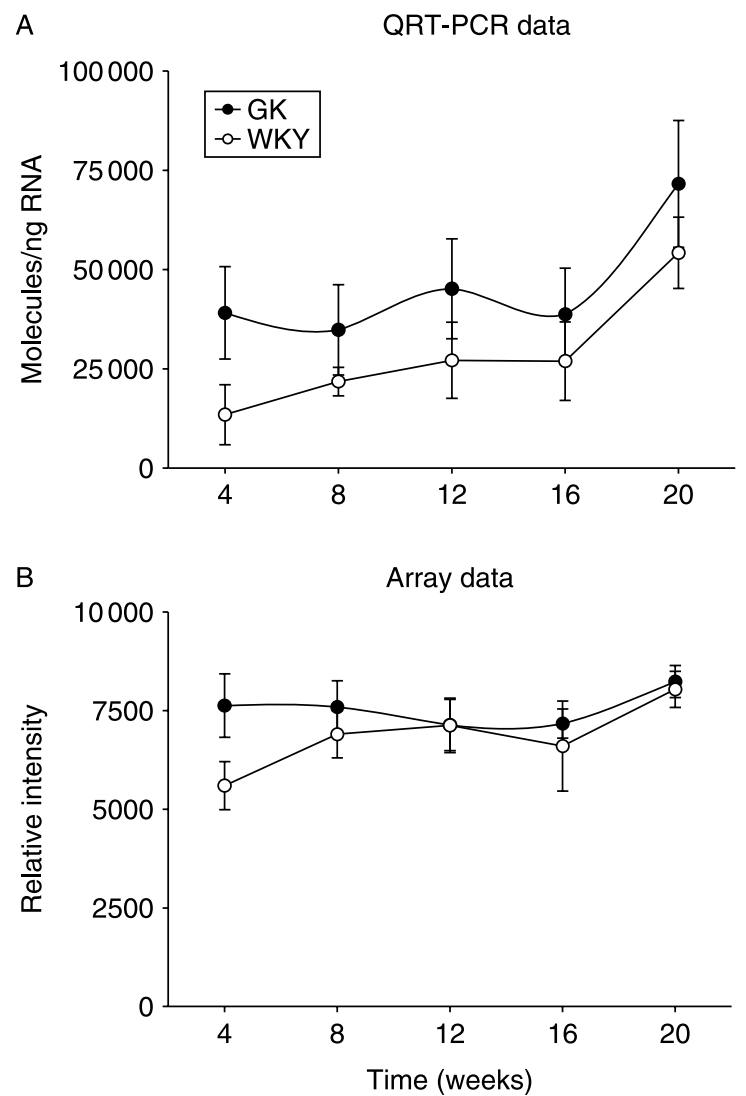

Figure 11 Expression of PCk1 mRNA in liver from GK and WKY rats as a function of age measured by kinetic-based real-time quantitative RT-PCT (A) and by gene arrays (B). Symbols represent mean values from five animals and error bars 1 S.D. of the mean.

hepatocytes. Insulin effects on the liver are several, most of which are designed to promote storage and inhibit glucose release. In the liver, insulin activates hexokinase activity and inhibits glucose-6-phosphatase that 'captures' the glucose in the cells. Insulin stimulates both glycogen synthesis and lipogenesis while inhibiting gluconeogenesis and glycogen breakdown. Most of the remaining excess glucose that passes the liver into the general circulation is taken up by the musculature through the insulin-dependent transporter, GLUT4. To a great degree, insulin resistance in muscle involves the inability of the musculature to remove glucose from circulation. A consequence is that blood circulating to the liver remains rich in glucose and glucose continues to flow into the liver through GLUT2 (Bloomgarden 2003). Because water follows glucose into cells, the free glucose must be disposed of in some way. Besides energy generation, there are only two ways to dispose of the glucose: substrate-driven lipogenesis and glycogen synthesis. Lipogenesis leading to steatosis is common in human type 2 diabetes and excessive glycogen storage has also been reported (Vallance-Owen 1952, Clore et al. 1992, Lonardo et al. 2005).
In the present study, we compared the post-natal growth and development of GK rats with control WKY rats from 4 to 20 weeks of age. Although GK rats were derived from WKY, the two groups of animals are clearly different in body weights, with WKY being significantly heavier (Fig. 1). There is a substantial increase in the weight of the livers of both strains between 4 and 8 weeks of age, with slower increases throughout the remainder of the experimental period. However, the increases in the livers of the GK rats were proportionately larger than those in WKY (Fig. 2), despite the absence of excessive fat deposition in the GK livers as indicated by liver triglyceride concentrations. One distinct possibility to be examined in the future is that the proportionately larger weight of the GK livers is due to deposition of glycogen resulting from their chronic hyperglycemia, as illustrated in Fig. 3. It has previously been reported that with increasing age, GK rats develop $\beta$-cell failure (Portha 2005).

Plasma insulin concentrations (Fig. 4) tend to support this observation. By 8 weeks of age, the GK rats have higher circulating insulin, which is consistent with the hyperglycemia, but by 20 weeks insulin concentrations are higher in WKY than GK, suggesting that the $\beta$-cells of the GK are no longer capable of fully responding to the hyperglycemia.

We globally examined the gene expression in the livers of diabetic GK rats compared with control WKY rats as a function of disease progression from 4 to 20 weeks of age. Data mining yielded 395 probe sets out of more than 31000 on the chip (less than 1.3\%) that had at least a twofold difference in expression between the two strains for at least three ages. Based on an extensive review of the literature, we divided these probe sets into functional groups and examined these groups for genes whose expression can provide insights into the pathology. Gene arrays simply provide a snapshot of the concentration of mRNAs at one point in time. The results reflect the potential available at that time for the production of proteins. When used in a time series as we have done in this experiment, we are able to observe how that potential changes with disease development and progression. By design, the process by which we obtained RNA from the liver was as heterogeneous as possible, sampling all cell types. Thus, we cannot determine whether a particular message is from the various populations of hepatocytes, the vasculature of the liver, or the extensive population of immune cells that reside in the liver. Although we cannot directly ascertain whether steady-state concentrations of message are due to mRNA production or destruction, we can obtain insight from examining messages for proteins involved in functional processes that can either contribute to hyperglycemia or may reflect the impact of hyperglycemia on the liver.

The liver resides at the end of the hepatic portal system and normally receives about $70 \%$ of its blood supply as blood that has circulated first to the small intestine, which is both a source of nutrients and a potential source of infection. In the normal situation, $10-15 \%$ of the total blood volume is in the liver, with roughly $60 \%$ of that in the sinusoids. Because of its 
location and large blood volume, the liver is very important for systemic immune surveillance and protection. Liver macrophages, Kupffer cells, are very actively phagocytic. The liver also produces approximately half of the lymph formed in the body. Chronic inflammation is linked to the development of insulin resistance, and chronic hyperglycemia is also linked to chronic inflammation (Wellen \& Hotamisligil 2005). If systemic inflammation is present one would expect to observe it in the liver. The profiles presented in Fig. 6 illustrate the presence of chronic inflammation in GK rats. As part of the natural immune response to bacteria, there is a rapid systemic response to LPS, a carbohydrate-lipid structure expressed on their outer cell wall of gram-negative bacteria. CD14 is a protein expressed on the surface of many cells (especially macrophages) that serves as a coreceptor for LPS (Kubota et al. 2004). In addition, liver secretes into circulation a soluble form of CD14 that can confer LPS responsiveness to other tissues (Kubota et al. 2004). To some degree, LPS is always present in circulation if for no other reason than arising from gut bacteria. Hyperresponsiveness to LPS has been observed in both human and animal forms of type 2 diabetes (Yang et al. 1997, Cani et al. 2007, Amar et al. 2008). The profiles for CD14 indicate chronic high levels of message for CD14 in GK rats relative to WKY (Fig. 6A).

IFNs are protein cytokines produced by immune cells in response to a variety of 'foreign' agents, in particular doublestranded RNA. Relationships between high levels of IFNs and insulin resistance have been reported in both humans and animals (Mofredj et al. 2002, Li et al. 2005). High levels of IFNs can occur for a variety of reasons, ranging from infections such as hepatitis to interferon therapy. The relationship between elevated IFN and insulin resistance is not absolute. Rather, some investigators hypothesize that insulin resistance in response to IFN only occurs in type 2 diabetic-prone humans and animals (Mofredj et al. 2002, Li et al. 2005). Ifit1 and Iigp 1 are two IFN-induced, proinflammatory genes that are chronically far more highly expressed in the livers of GK rats than in the livers of control WKY animals (Fig. 6B and C). CXCL14 is a secreted protein involved in immunoregulatory and inflammatory processes (Nara et al. 2007), which is also chronically upregulated in the livers of GK rats (Fig. 6D). CXCL14 is involved in attracting dendritic cells and it has been reported that this chemokine may be specific for Cd14-positive cells (Meuter et al. 2007), which is relevant, given the high expression of Cd14 in the livers of GK rats discussed earlier. Just as there are processes that promote inflammation, there are those that inhibit inflammatory signaling. SOCS2, which is a member of a family of suppressor of cytokine signaling proteins, is more highly expressed in the livers of WKY animals (Supplementary Table 2). SOCS2 interacts with another negative regulator of cytokine signaling, CIS (Uyttendaele et al. 2007), which also has a lower expression profile in GK (Supplementary Table 2). The final example is Traf6, which is more highly expressed in the livers of WKY rats (Supplementary Table 2). TRAF6 has been shown to interfere with the
IL1B-induced signaling pathway involved in inflammation (Matsumura et al. 2004).

Clearly, defects in insulin signaling are involved in some types of human type 2 diabetes. With respect to the GK rat model of type 2 diabetes, the results are rather mixed. In this experiment, we observed a number of differences in the expression of genes in the livers of GK rats versus WKY, which could significantly contribute to the diabetic phenotype. Four examples of differences in expression profiles between GK and WKY are shown in Fig. 7. PI 3-kinase, regulatory subunit, polypeptide 1 (p85 alpha) (Pik3r1) is a regulatory subunit that interacts with a type 1a catalytic subunit. This catalytic subunit is directly downstream from IRS proteins in the insulin signaling pathway. Decreased expression of Pik3r1 suggests a possible decrease in insulin signaling at the level of PI3-kinase (Fig. 7A). Sandu et al. (2000) reported that in vascular smooth muscle, the signal transfer between IRS1 and PIK3R1 is disrupted in GK rats (Almind et al. 2002, Ueki et al. 2003, Matsumura et al. 2004) However, they attributed this to a close downstream step involving myosin-bound phosphatase (MYPT).

On the chip, there are probe sets for Ppp1r12a, Ppp1r12b, Ppp1r16a, Mypt4, and Mypt5. However, a review of the results for these probe sets showed that there was no difference between GK and WKY for any MYPT isoforms. PI 3-kinases are a family of enzymes that are involved in regulating a large number of diverse processes in virtually all tissues by generating lipid second messengers. There are three general types of PIK3R1s. The aforementioned PIK3R1 is in a type 1a pathway, which is activated by tyrosine kinase-associated receptors (Almind et al. 2002, Ueki et al. 2003, Matsumura et al. 2004). The second gene in Fig. 7 is Pik3c3, also known as $V p s 34$, which is a catalytic subunit in a type 3 pathway (Almind et al. 2002, Ueki et al. 2003, Matsumura et al. 2004). PIK3C3 does not interact with PIK3R1, but rather interacts with the p150 regulatory subunit. The profile for Pik3c3 showed that the expression for this gene was considerably higher in WKY relative to GK (Fig. 7B). This result would suggest that in the livers of GK rats, there is reduction in the intracellular movement of membrane proteins because type 3 pathways are involved in vesicle trafficking. Pftk1 codes for a cyclin-dependent kinase (Shu et al. 2007), which is chronically much more highly expressed in the livers of WKY than GK (Fig. 7C). This result suggests that cell proliferation is reduced in the livers of GK rats relative to WKY. As discussed above, one of the functional themes that distinguish GK from WKY is inflammation. In exploring possible relationships, we found that IFNs, whether pathologically or therapeutically elevated, inhibit cell proliferation in the liver (Degenhardt et al. 2006) and may relate to the lower expression of Pftk1 in the livers from GK rats. The last example presented in the signal transduction group is Mtmr 7 , which is a lipid phosphatase that removes phosphate groups on the phosphorylated inositol head group (Mochizuki \& Majerus 2003). This phosphatase is chronically upregulated in the livers of GK rats when compared with WKY (Fig. 7D). 
This is particularly interesting because Mtmr7 is counterregulatory to $\mathrm{Pik3c3}$, which is much lower in GK relative to WKY (Fig. 7B). Together, the two results indicate that there is much lower activity in GK relative to WKY rats in this pathway involved in regulating vesicle trafficking. Other genes of interest in this functional category include Igfbp 1 and Dyrk1a (Supplementary Table 2). IGFBP1 is a hepatocytederived secreted protein. The primary role of IGFBP1 appears to be binding and sequestering insulin-like growth factor 1 (IGF1), thus reducing its availability (Degenhardt et al. 2006). IGF1 is structurally and functionally related to insulin and contributes to glucose disposal from circulation. The expression of Igfbp 1 is substantially higher in the livers of GK relative to WKY rats. Data are available demonstrating that elevating circulating IGFBP1 elevates the circulating glucose concentration (Degenhardt et al. 2006).

Dual-specificity tyrosine-(Y)-phosphorylation regulated kinase 2 (DYRK2) is a cytoplasmic member of a family of priming kinases. These enzymes phosphorylate residues important to the phosphorylation of adjacent serine residues. Although at present the role of DYRK2 in the liver is not well understood, the members of the DYRK family have been shown to play an important role in the activation/inactivation of both the translation initiation factor EIF2B and glycogen synthase kinase 3 beta (GSK3B; Cohen \& Goedert 2004, Wilson et al. 2005). Insulin is a prominent regulator of the activity of both EIF2B and GSK3B, which are keys to the regulation of protein and glycogen synthesis respectively. Therefore, it may be important that in WKY there is a steady increase in the expression of Dyrk2 with age, but that this increase does not occur in the livers of GK rats.

Gene arrays only provide mRNA concentrations for a large number of genes at the time of killing. However, since the expressions of specific genes are involved in producing proteins, the results do provide indications concerning the availability of groups of proteins. For this reason, we have constructed a functional group that includes transcription and translation, and present examples of a number of differentially regulated genes in this category that may have relevance to the diabetic phenotype. STAT3 is a member of the family of transcription factors known as 'signal transducer and activator of transcription'. STATs are activated by tyrosine phosphorylation followed by dimerization and translocation into the nucleus where they bind to cognate sites in the promoter regions of responsive genes (Maritano et al. 2004). STAT3 mediates quite diverse functions in different tissues. In vascular endothelial cells, it mediates the expression of genes that protect against inflammation induced by LPS (Kano et al. 2003, Maritano et al. 2004). In hepatocytes, it mediates the aspects of the acute phase response promoting the expression of both pro- and anti-inflammatory proteins (Maritano et al. 2004). Similarly, its effect on the various immune cells is both pro- and anti-inflammatory being involved in both IL6 and IL10 signaling. Because of the heterogeneous population of cells contributing to our array results, it is difficult to interpret the results for this gene except that regulation of inflammation is relevant in all cell types. Therefore, it is significant in that the expression of Stat3 is chronically much lower in GK rats when compared with WKY (Fig. 8A).

A second example is provided by Crym, which is relevant to thyroid hormone function. Thyroid hormone receptor, like the glucocorticoid receptor, is a member of the nuclear hormone receptor superfamily and participates in the regulation of a subset of genes. The activating ligand is 3,3',5-triiodothyronine $\left(\mathrm{T}_{3}\right)$. CRYM is a NADPH-dependent cytosolic $\mathrm{T}_{3}$-binding protein that participates in the regulation of the availability of $\mathrm{T}_{3}$ to thyroid hormone receptor (Suzuki et al. 2007). Figure $8 \mathrm{~B}$ demonstrates that the message for Crym is chronically higher in the liver of WKY when compared with GK rats. Given the role of thyroid hormone in regulating the expression of genes involved in energy metabolism, this difference in gene expression may be relevant to diabetes in GK rats.

Another transcription factor chronically elevated in GK samples is CREBL2. AMP-activated protein kinase (AMPK) is a major sensor of cellular energy charge. The general role of AMPK is to enhance ATP production and to reduce ATP utilization. In the liver, phosphorylation of targets by AMPK inhibits lipogenesis and gluconeogenesis while promoting the oxidation of fatty acids. Both enzymes such as HMG-CoA reductase (the rate-limiting enzyme in cholesterol biosynthesis) and transcription factors are targets of AMPK (Thomson et al. 2008). CREBL2 is a transcription factor whose activity is influenced by AMPK and several other kinases. CREBL2 is a member of the CREB family of transcription factors that bind to cAMP-response element (CRE) in the promoter regions of responsive genes (Thomson et al. 2008). Phosphorylation of CREBL2 activates it and promotes its translocation into the nucleus and binding to CREs. Thus, it may be relevant that this transcription factor has chronically higher expression in the livers from GK rats relative to WKY. In general, this transcription factor would be expected to participate in the inhibition of gluconeogenesis in the liver. Based on studies treating GK rats with troglitazone, O'Rourke et al. (1997) concluded that gluconeogenesis in the liver contributes to hyperglycemia in this model, much as it appears to do in some human type 2 diabetics. The chronically elevated levels of Crebl2 seen here appear contrary to that conclusion. Cellular repressor of E1A-stimulated genes 1 (Creg 1) was placed in this functional group instead of signal transduction because it influences the expression of a distinct set of genes (Tsuchiya et al. 2004). The expression profiles of this gene in the GK and WKY livers suggest that the observed difference may be a consequence of hyperglycemia. Specifically, there is not much difference at 4 weeks of age, but with increasing age GK becomes increasingly higher than WKY, with a pattern similar to the increased hyperglycemia (Fig. 8C). CREG1 is a secreted phosphoglycoprotein that represses E1A-stimulated genes. These genes stimulate cell division and inhibit differentiation. Specifically, during the cell cycle, they promote the transition from G1- to S-phase and the start of 
DNA synthesis (Veal et al. 1998). The last gene in this group is Rabl3 (Fig. 8D). Following translation, a membrane protein must be both folded properly and inserted into the membrane. Rabl3 is a member of the RAB family of GTPases, which is involved in membrane trafficking to tight junctions in hepatocytes (Wilton \& Matthews 1996). It has been reported that LPS disrupt tight junction in the liver (Sheth et al. 2007). The chronic higher expression of Rabl3 in the livers of GK compared with WKY rats may reflect chronic repair of the damage caused by chronic inflammation. Another example in this functional category is nocturnin (Supplementary Table 2). Nocturnin, also known as CCR 4 carbon catabolite repression 4 -like (S. cerevisiae) (Ccrn $4 l)$, is a deadenylase and as such promotes the degradation of mRNAs. Nocturnin is important to regulating the concentration of messages for proteins involved in energy metabolism (Green et al. 2007). What is interesting about this gene is that its expression has a distinct circadian pattern with the highest expression early in the active feeding period of these nocturnal animals (Green et al. 2007) It is therefore important that these animals were killed early in the inactive rest period when the expression of nocturnin should be at its lowest point. The expression of this gene is consistently higher in the livers of GK when compared with WKY rats. Because we only measured expression at one time within the 24-h cycle, we cannot determine whether the difference between GK and WKY reflects a disturbance in circadian rhythms in GK rats, which itself has been shown to contribute to insulin resistance and metabolic syndrome, or whether the difference occurs throughout the circadian rhythm. However, it is relevant that knocking out nocturnin in mice confers resistance to diet-induced hepatic steatosis and obesity (Green et al. 2007).

Part of our criteria for identifying genes that are different between GK and WKY was a twofold difference in at least three out of five time points. PCK1, the rate-limiting enzyme for gluconeogenesis, clearly did not meet this criterion. However, because of the importance of this enzyme to glucose homeostasis, we directly looked at the probe set for PCK1. The array results showed that only at 4 weeks was the expression of Pck1 mRNA substantially higher in GK relative to WKY. Additionally, we measured the expression of Pck1 mRNA using quantitative fluorescence-based real-time RT-PCR. The results confirmed the substantially higher expression in GK at 4 weeks. However, smaller differences at three other times also tested significant at a much lower probability. Thus, our data suggest that excessive gluconeogenesis may only contribute significantly to the less than twofold elevation in glucose seen in GK rats at the earliest time point (4 weeks), but not to the several fold elevation in glucose seen at later time. Therefore, the results suggest that impaired glucose disposal is most likely the basis of the chronic hyperglycemia at the later ages.

Chronic hyperglycemia due to insulin resistance places the liver in a situation where its blood supply is chronically rich in glucose. In this situation, large amounts of glucose flow into the liver through the insulin-independent GLUT2. The two primary ways of disposing of this excess glucose are substratedriven lipid synthesis and glycogen synthesis. It is well documented that many type 2 diabetics develop steatosis (Lonardo et al. 2005). One of the most populated functional groups we found was lipid metabolism. The four examples from the lipid metabolism group shown in Fig. 9 suggest that alteration in lipid metabolism in the liver is a major consequence of the chronic hyperglycemia in GK rats. The first of these genes is Ephx2. As shown in Fig. 9A, the expression of this gene is very low in GK relative to WKY. This is important because a defect in this gene is associated with a type of hereditary hyperlipidemia, familial hypercholesterolemia, insulin resistance, and hypertension, suggesting that the activity of this gene is important for the normal control of cholesterol (Sato et al. 2004, Lee et al. 2006) Similarly, the expression in Sult1c2, which is involved in the degradation of bile acids through sulfation and therefore participates in cholesterol metabolism (Vemula et al. 2004), is much lower in GK relative to WKY (Fig. 9B). However, in this case, the expression of the gene increases with age in the WKY but remains low in GK.

By contrast, citrate lyase beta like $(C l y b l)$, which is involved in fatty acid biosynthesis (Morikawa et al. 2001), becomes progressively higher in GK relative to WKY with age due to the decline of this enzyme in WKY (Supplementary Table 2). Consistent with a picture of elevated lipid biosynthesis in GK is the higher amount of mRNA for ELOVL family member 6 (Elo2), which is involved in the elongation of long-chain fatty acids (Kobayashi et al. 2007), and acyl-CoA synthetase medium-chain family member 2 (Acsm2), which is involved in the production of medium-chain acyl-CoA (Lindner et al. 2006; Supplementary Table 2). The expression of both Abca1 and Apoa 2 are much higher in GK at all ages (Fig. 9C and D). ABCA1 functions as a cholesterol efflux pump in the cellular lipid removal pathway, and is rate limiting in HDL particle assembly (Tanaka et al. 2008) while APOA2 is the second most abundant apolipoprotein in HDL (Weng \& Breslow 1996). The elevations in message for these two genes suggest elevation in circulating HDL. An elevation in HDL may help explain the resistance of GK rats to arthrosclerosis reported by Ragolia et al. (2004). Adding to the picture of resistance to arthrosclerosis in GK rats is the lower expression of Apobec1 (Supplementary Table 2), which is the catalytic component of the Apob mRNA editing enzyme complex. Lowering the expression of this gene should lower LDL (Dutta et al. 2003). Fabp 7 is consistently lower in GK relative to WKY, while the expression of Fabp2 (Supplementary Table 2) is elevated in GK relative to WKY. These differences are interesting because dysregulation of fatty acid-binding proteins is thought to provide a link between lipid metabolism and chronic inflammation, which often accompanies type 2 diabetes in humans (Linton \& Fazio 2003).

More than half of the 306 identified genes are encompassed by the functional groups discussed earlier. However, there are genes not in these groups that may provide insights into both 
the cause and the consequences of diabetes in GK rats. Some examples are provided here. The first two are Blvrb and Gp $x 2$, both of which are more highly expressed in the livers of GK relative to WKY (Fig. 10A and B). These two antioxidant enzymes are markers of oxidative stress that is attendant on diabetes (Wang \& Ortiz de Montellano 2003, Yan \& Chen 2006). Another, Cyp3a25, whose expression is influenced by both $\mathrm{GH}$ and testosterone (Kawai et al. 2000) shows an age-related progressive increase in the expression in WKY that does occur in GK (Supplementary Table 2). One consequence of chronic hyperglycemia on the liver is to compromise liver function. The difference in the expression of Cyp3a25 most likely reflects the impact of diabetes on liver function. Similarly, the higher expression of Uqcrb, a mitochondrial respiratory chain enzyme is probably consequential and may be related to oxidative stress (Supplementary Table 2). RESP18 is a protein, the glycosylated form of which is secreted. This gene seems to be involved in regulating systemic stress responses (Bloomquist et al. 1994). The higher expression in GK relative to WKY probably reflects a consequence of diabetes (Fig. 10C). Mup5 is a member of the lipocalin family whose expression is highly androgen dependent and has been reported to only be expressed at about 40 days of age in male rats (Rowland et al. 2005). Consistent with that observation, our data show that the message for this protein is virtually absent in the livers of both strains at 4 weeks but appears in both at 8 weeks (Fig. 10D). However, the expression in the livers of WKY declines with age while remaining rather high in GK. Lipocalins bind and transport hydrophobic molecules and the difference between the two strains may reflect the altered lipid metabolism in GK rats discussed earlier.

The results of this study strongly support the hypothesis that chronic inflammation causally contributes to the development of diabetes in GK rats just as it does in a subset of humans with type 2 diabetes. Continued testing of this hypothesis will involve the analysis of other tissues from these animals as well as physiological measurements relevant to inflammation. Each animal in this study had a unique identifier and all samples are indexed to this identifier. Supplementary Table 1, which provides the raw data for each individual probe set identified as differentially regulated in these livers, also provides an animal code for each individual chip. It is our intent to provide this code information for all reported measurements in future publications from our ongoing studies, whether they be gene arrays, biochemical, or physiological. From a systems biology perspective, this will make these studies most useful to other investigators.

\section{Declaration of interest}

There is no conflict of interest that could be perceived as prejudicing the impartiality of the research reported.

\section{Funding}

This work was supported by funds from the UB Pfizer Strategic Alliance.

\section{Acknowledgements}

This dataset was developed at the Children's National Medical Center under the auspices of a grant from the National Heart, Lung, and Blood Institute (NHLBI)/NIH Programs in Genomic Applications HL 66614 (Eric P Hoffman, PI). We would like to acknowledge the technical assistance of Ms Nancy Pyszczynski and Ms Suzette Mis.

\section{References}

Almind K, Delahaye L, Hansen T, Van Obberghen E, Pedersen O \& Kahn CR 2002 Characterization of the Met326lle variant of phosphatidylinositol 3-kinase p85a. PNAS 99 2124-2128.

Almon RR, DuBois DC, Jin JY \& Jusko WJ 2005a Temporal profiling of the transcriptional basis for the development of corticosteroid-induced insulin resistance in rat muscle. Journal of Endocrinology $184219-232$.

Almon RR, Lai W, DuBois DC \& Jusko WJ 2005 b Corticosteroid-regulated genes in rat kidney: mining time series array data. American Journal of Physiology. Endocrinology and Metabolism 289 E870-E882.

Almon RR, DuBois DC \& Jusko WJ 2007a A microarray analysis of the temporal response of liver to methylprednisolone: a comparative analysis of two dosing regimens. Endocrinology 148 2209-2225.

Almon RR, DuBois DC, Yao Z, Hoffman EP, Ghimbovschi S \& Jusko WJ $2007 b$ Microarray analysis of the temporal response of skeletal muscle to methylprednisolone: comparative analysis of two dosing regimens. Physiological Genomics 30 282-299.

Amar J, Burcelin R, Ruidavets JB, Cani PD, Fauvel J, Alessi MC, Chamontin B \& Ferrieres J 2008 Energy intake is associated with endotoxemia. American Journal of Clinical Nutrition 87 1219-1223.

Bauer M, Hamm AC, Bonaus M, Jacob A, Jaekel J, Schorle H, Pankratz MJ \& Katzenberger JD 2004 Starvation response in mouse liver shows strong correlation with life-span-prolonging processes. Physiological Genomics 17 230-244.

Bloomgarden ZT 2003 Pharmacologic treatment of type 2 diabetes. Diabetes Care 26 526-533.

Bloomquist BT, Darlington DN, Mueller GP, Mains RE \& Eipper BA 1994 Regulated endocrine-specific protein-18: a short-lived novel glucocorticoid-regulated endocrine protein. Endocrinology 135 2714-2722.

Boehm U, Guethlein L, Klamp T, Ozbek K, Schaub A, Futterer A, Pfeffer K \& Howard JC 1998 Two families of GTPases dominate the complex cellular responses to INF-gamma. Journal of Immunology 161 6715-6723.

Cani PD, Amar J, Iglesias MA, Poggi M, Knauf C, Bastelica D, Neyrinck AM, Fava F, Tuohy KM, Chabo C et al. 2007 Metabolic endotoxemia intiates obesity and insulin resistance. Diabetes 56 1761-1772.

De Caterina R \& Basta G 2001 n-3 Fatty acids and the inflammatory response-biological background. European Heart Journal 3 D42-D49.

Clore JN, Post EP, Bailey DJ, Nestler JE \& Blackard WG 1992 Evidence for increased liver glycogen in patients with noninsulin-dependent diabetes mellitus. Journal of Clinical Endocrinology and Metabolism 74 660-666.

Cohen P \& Goedert M 2004 GSK3 inhibitors: development and therapeutic potential. Nature Reviews. Drug Discovery 3 479-487.

Degenhardt T, Matilainen M, Herzig K, Dunlop TW \& Carlberg C 2006 The insulin-like growth factor-binding protein 1 gene is a primary target of peroxisome proliferator-activated receptors. Journal of Biological Chemistry 281 39607-39619.

Dutta R, Singh U, Li TB, Fornage M \& Teng BB 2003 Hepatic gene expression profiling reveals perturbed calcium signaling in a mouse model lacking both LDL receptor and apobec1 genes. Atherosclerosis 169 51-62. 
Gastaldelli A, Baldi S, Pettiti E, Camastra S, Natali A, Landau BR \& Ferrannini E 2000 Influence of obesity and type 2 diabetes on gluconeogenesis and glucose output in humans. Diabetes 49 1367-1373.

Green CB, Douris N, Kojima S, Strayer CA, Fogerty J, Lourim D, Keller SR \& Besharse JC 2007 Loss of nocturnin, a circadian deadenylase, confers resistance to hepatic steatosis and diet-induced obesity. PNAS.

Hundal RS, Krssak M, Dufour S, Laurent D, Lebon V, Chandramouli V, Inzucchi SE, Schumann WC, Petersen KF, Landau BR et al. 2000 Mechanism by which metaformin reduces glucose production in type 2 diabetes. Diabetes 49 2063-2069.

Kano A, Wolfgang MJ, Gao Q, Jacoby J, Chai G, Hansen W, Iwamoto Y, Pober JS, Flavell RA \& Fu X 2003 Endothelial cells require STAT3 for protectin against endotoxin-induced inflammation. Journal of Experimental Medicine 198 1517-1525.

Kawai M, Bandiera SM, Chang TKH \& Bellward GD 2000 Growth hormone regulation and developmental expression of rat hepatic CYP3A18, CYP3A9, and CYP3A2. Biochemical Pharmacology 59 1277-1287.

Kobayashi T, Zadravec D \& Jacobsson A 2007 ELOVL2 overexpression enhances triacylglycerol syntesis in 3T3-L1 and F442A cells. FEBS Letters $\mathbf{5 8 1} 3157-3163$

Kubota K, Kim JY, Sawada A, Tokimasa S, Fujisaki H, Matsuda-Hashii Y, Ozono K \& Hara J 2004 LRRC8 involved in B cell development belongs to a novel family of leucine-rich repeat proteins. FEBS Letters 564 147-152.

Lee CR, North KE, Bray MS, Fornage M, Seubert JM, Newman JW, Hammock BD, Couper DJ, Heiss G \& Zeldin DC 2006 Genetic variation in soluble epoxide hydrolase (EPHX2) and risk of coronary heart disease: the aterosclerosis risk communities (ARIC) study. Human Molecular Genetics 15 1640-1649.

Li Z, Soloski MJ \& Diehl AM 2005 Dietary factors alter hepatic innate immune system in mice with nonalcholic fatty liver disease. Hepatology $\mathbf{4 2}$ 880-885

Lindner I, Rubin D, Helwig U, Nitz I, Hampe J, Schreiver S, Schrezenmeir J \& Doring F 2006 The L513S polymorphism in meium-chain acyl-CoA synthetase 2 (MACS2) is associated with risk factors of the metabolic syndrome in a Caucasian study population. Molecular Nutrition $\&$ Food Research 50 270-274.

Linton MF \& Fazio S 2003 Macrphages, inflammation, and atherosclerosis. International Journal of Obesity 27 S35-S40.

Lonardo A, Lombardini S, Ricchi M, Scaglioni F \& Loria P 2005 Hepatic steatosis and insulin resistance. Alimentary Pharmacology and Therapeutics 22 $64-70$.

Maritano D, Surgue ML, Tininini S, Dewilde S, Strobl B, Fu XP, Murray-Tait V, Chiarle R \& Poli V 2004 The STAT3 isoforms A and B have unique and specific functions. Nature Immunology 5 401-409.

Matsumoto M, Ogawa W, Akimoto K, Inoue H, Miyake K, Furukawa K, Hayashi Y, Iguchi H, Matsuki Y, Hiramatsu R et al. 2003 PKC in liver mediates insulin-induced SREBP-1c expression and determines both hepatic lipid content and overall insulin sensitivity. Journal of Clinical Investigation $112935-944$.

Matsumura T, Semba K, Azuma S, Ikawa S, Gohda J, Akiyama T \& Inoue J 2004 TIFAB inhibits TIFA, TRAF-interacting protein with a forkhead associated domain. Biochemical and Biophysical Research Communications 317 230-234.

Meuter S, Schaerli P, Roos RS, Brandau O, Bosl MR, von Andrian UH \& Moser B 2007 Murine CXCL1 is dispensable for dendritic cell function and localization within peripheral tissues. Molecular and Cellular Biology 27 983-992.

Mochizuki Y \& Majerus PW 2003 Characterization of myotubularin-related protein 7 and its binding partner, myotubularin-related protein 9. PNAS $1009768-9773$.

Mofredj A, Howaizi M, Grasset D, Licht H, Loison S, Devergie B, Demontis R \& Cadranel J-F 2002 Diabetes mellitus during interferon therapy for chronic viral hepatitis. Digestive Diseases and Sciences 47 1649-1654.

Morikawa J, Nishimura Y, Uchida A \& Tanaka T 2001 Molecular cloning of novel mouse and human putative citrate lyase B-subunit. Biochemical and Biophysical Research Communications 289 1282-1286.
Nara N, Nakayama Y, Okamoto S, Tamura H, Kiyono M, Muraoka M, Tanaka K, Taya C, Shitara H, Ishii R et al. 2007 Disruption of CXC motif chemokine ligand-14 in mice ameliorates obesity-induced insulin resistance. Journal of Biological Chemistry 282 30794-30803.

Ogawa S, Lozach J, Benner C, Pascual G, Tangirala RK, Westin S, Hoffmann A, Subramaniam S, David M, Rosenfeld MG et al. 2005 Molecular determinants of crosstalk between nuclear receptors and toll-like receptors. Cell 122 707-721.

O'Rourke CM, Davis JA, Saltiel AR \& Cornicelli JA 1997 Metabolic effects of troglitazone in the Goto-Kakizaki rat, a non-obese and normolipidemic rodent model of non-insulin dependent diabetes mellitus. Metabolism $\mathbf{4 6}$ 192-196.

Portha B 2005 Programmed disorders of B-cell development and function as one cause for type 2 diabetes? The GK rat paradigm Diabetes/Metabolism Research and Reviews 21 495-504.

Prando R, Cheli V, Melga P, Giusti R, Ciuchi E \& Odetti P 1998 Is type 2 diabetes a different disease in obese and nonobese patients? Diabetes Care $\mathbf{2 1}$ $1680-1685$.

Ragolia L, Plaia T, Koutrouby TB \& Maesaka JK 2004 Inhibition of cell cycle progression and migration of vascular smooth muscle cells by prostaglandin D2 synthase: resistance in diabetic Goto-Kakizaki rats. American Journal of Physiology. Cell Physiology 287 C1273-C1281.

Rowland JE, Lichanska AM, Kerr LM, White M, d'Aniello EM, Maher SL, Brown R, Teasdale RD, Noakes PG \& Waters MJ 2005 In vivo analysis of growth hormone receptor signaling domains and their associated transcripts. Molecular and Cellular Biology 25 66-77.

Sato K, Emi M, Ezura Y, Fujita Y, Ishigami T, Xin Y, Wu L \& Larrinaga-Shum S 2004 Soluble epoxide hyrolase variant (Glu287Arg) modifies plasma total cholesterol and triglyceride phenotype in familial hypercholesterolemia: intrafamilial association study in an eight-generation hyperlipidemic kindred. Journal of Human Genetics 47 29-34.

Schiller MR, Mains RE \& Eipper BA 1995 A neuroendocrine-specific protein localized to the endoplasmic reticulum by distal degradation. Journal of Biological Chemistry 270 26129-26138.

Sedova L, Seda O, Kazdova L, Chylikova B, Hamet P, Tremblay J, Kren V \& Krenova D 2007 Sucrose feeding during pregnancy and lactation elicts distinct metabolic response in offspring of an inbred genetic model of metabolic syndrome. American Journal of Physiology. Endocrinology and Metabolism 292 E1318-E1324.

Sheth P, Santos ND, Seth A, LaRusso NF \& Rao RK 2007 Lipoplysaccharide disrupts tight junctions in cholangiocyte monolayers by a c-Src-, TLR4-, and LBP-dependent mechanism. American Journal of Physiology. Gastrointestinal and Liver Physiology 293 G308-G318.

Shu F, Lu S, Qin Y, Ma X, Wang X, Peng X, Luo Y, Xu B, Sun X \& Wu J 2007 Functional characterization of human PFTK1 as a cyclin-dependent kinase. PNAS 104 9248-9253.

Suzuki S, Mori J \& Hashizume K 2007 mu-Crystallin, a NADPH-dependent $\mathrm{t}(3)$-binding protein in cytosol. Trends in Endocrinology and Metabolism 18 286-289.

Tanaka Y, Aleksunes LM, Yeager RL, Gyamfi MA, Esterly N, Guo GL \& Klaassen CD 2008 NF-E2-related factor 2 inhibits lipid accumulation and oxidative stress in mice fed a high-fat diet. Journal of Pharmacology and Experimental Therapeutics 325 655-664.

Thomson DM, Herway ST, Fillmore N, Kim H, Brown JD, Barrow JR \& Winder WW 2008 AMP-activated protein kinase phosphorylates transcription factors of the CREB family. Journal of Applied Physiology 104 429-438.

Tsuchiya T, Dhahbi JM, Cui X, Mote PL, Bartke A \& Spindler SR 2004 Additive regulation of hepatic gene expression by dwarfism and caloric restriction. Physiological Genomics 17 307-315.

Ueki K, Fruman DA, Yballe CM, Fasshauer M, Klein J, Asano T, Cantley LC \& Kahn CR 2003 Positive and negative roles of p85a and p85B regulatory subunits of phospoinositide 3-kinase in insulin signaling. Journal of Biological Chemistry 278 48453-48466.

Uyttendaele I, Lemmens I, Verhee A, De Smet A-S, Vandekerckhove J, Lavens D, Peelman F \& Tavernier J 2007 Mammalian protein-protein interaction trap (MAPPIT) analysis of STAT5, CIS, and SOCS2 interactions with the growth hormone receptor. Molecular Endocrinology 21 2821-2831. 
Vallance-Owen J 1952 Liver glycogen in diabetes mellitus. Journal of Clinical Pathology 5 42-53.

Veal E, Eisenstein M, Tseng ZH \& Gill G 1998 A cellular repressor of E1Astimulated genes that inhibits activation by E2F. Molecular and Cellular Biology 18 5032-5041.

Vemula M, Berthiaume F, Jayaraman A \& Yarmush ML 2004 Expression profiling analysis of the metabolic and inflammatory changes following burn injury in rats. Physiological Genomics 18 87-98.

Wang J \& Ortiz de Montellano PR 2003 The binding sites on human heme oxygenase-1 for cytochrome $\mathrm{P} 450$ reductase and biliberdin reductase. Journal of Biological Chemistry 278 20069-20076.

Wellen KE \& Hotamisligil GS 2005 Inflammation, stress, and diabetes. Journal of Clinical Investigation 115 1111-1119.

Weng W \& Breslow JL 1996 Dramatically decreased high density lipoprotein cholesterol, increased remnant clearance, and insulin hypersensitivity in apolipoprotein A-II knockout mice suggest a complex role for apolipoprotein in atherosclerosis susceptibility. PNAS $9314788-14794$.
Wilson WA, Skurat AV, Probst B, Paoli-Roach A, Roach PJ \& Rutter J 2005 Control of mammalian glycogen synthase by PAS kinase. PNAS 102 16596-16601.

Wilton JC \& Matthews GM 1996 Polarised membrane traffic in hepatocytes. BioEssays 18 229-236.

Yan W \& Chen X 2006 GPX2, a direct target of p63, inhibits oxidative stressinduced apoptosis in a p53-dependent manner. Journal of Biological Chemistry $2817856-7862$

Yang SQ, Lin HZ, Lane MD, Clemens M \& Diehl AM 1997 Obesity increases sensitivity to endotoxin liver injury: implications for the pathogenesis of steatohepatitis. PNAS 94 2557-2562.

Received in final form 18 November 2008

Accepted 3 December 2008

Made available online as an Accepted Preprint

9 December 2008 